\title{
COMMUNICATIONS
}

\section{TOXOPLASMOSIS IN THE ADULT*}

\author{
BY \\ SIR STEWART'DUKE-ELDER, NORMAN ASHTON, \\ AND M. 'BRIHAYE-VAN GEERTRUYDEN
}

From the Institute of Ophthalmology, University of London

Toxoplasmosis as a congenital infection is now a well-established clinical entity, but our knowledge of toxoplasmosis acquired in childhood or adult life is much less complete. It is known, however, that such infections may occur, and several cases without ocular involvement have been reported (Pinkerton and Weinman, 1940 ; Pinkerton and Henderson, 1941 ; Guimarães, 1943 ; Syverton and Slavin, 1946 ; Kean and Grocott, 1947 ; Noetzel, 1951), and Sabin (1942) described an acquired form of the disease in older children. Most of the cases of acquired toxoplasmosis in man have not shown ocular involvement, and Sabin (1950) and Sabin and others (1952), have emphasized the fact that there is as yet no satisfactory evidence that the organism can cause a chorio-retinitis through a post-natally acquired infection. Although he did not deny its possible occurrence, Sabin (1950) pointed out that the fully developed and post-natal nervous tissues are unquestionably less susceptible to the pathogenic effects of toxoplasma than are those of the embryonic nervous system.

Nevertheless, there are in the literature a few cases of toxoplasmosis with chorio-retinitis where acquired infection in childhood or adult life has been suspected. Six cases of chorio-retinitis associated with toxoplasma were reported by Vail, Strong, and Stephenson (1943) and, in their view, one patient aged 16 years, undoubtedly had an acquired infection. Magnusson (1951) reported seven cases of adult infection (two of whom had been infected during their work in the bacteriological laboratory); one of the patients had acute chorio-retinitis and the serological tests supported the diagnosis of toxoplasmosis. Wising (1952) described the case of a female aged 31 years, who presented with lymphadenopathy, pyrexia, and an acute juxtamacular chorio-retinitis in the left eye ; the clinical diagnosis of toxoplasmosis was confirmed by strongly positive dye and complement-fixation tests. Rieger $(1951,1952)$, reported fourteen cases of central exudative retinitis in adults, which, on the basis of skin and serum tests, were considered to be due to acquired toxoplasmosis.

It is not yet established whether a state of chronic toxoplasmosis can exist in man, as it may in animals. Although the fact that mothers who give birth to toxoplasma-infected diseased infants do not themselves show clinical

* Received for publication April 13, 1953 
evidence of the disease proves that the condition may occur in a latent of sub-clinical form, the normality of all subsequent pregnancies argues against a prolonged chronic infection. On the other hand, a case reported by Boh and Koch (1951), suggests that toxoplasma may persist in the body in a latent: form for many years. This patient, a female aged 22 years, developed acute chorio-retinitis, the dye tests were positive, and cerebral calcification was present ; this, according to the studies of Johnson, Fried, Broaddus, an Lamfrom (1946), probably indicates a congenital infection or one acquire in early childhood. Furthermore, an account of an interesting familys showing a syndrome of chronic meningo-encephalitis, chorio-retinitis, rash $\vec{\emptyset}$ nerve-deafness, and eosinophilia due to toxoplasmosis, was reported by Campbell and Clifton (1950); it was apparent that the infection had re mained in a subacute form in three, and possibly four, members of the family. for many years. Kean and Grocott (1947) found an aggregation of parasites probably toxoplasma, in the cardiac muscle of a healthy Negro who comi mitted suicide, and Plaut (1946) also demonstrated toxoplasma in the myocardium in a child and in an adult. It would appear possible, therefore? that an active chorio-retinitis in an adult may be due not only to a recently acquired infection, but also to an exacerbation of an old infection eithe post-natally or congenitally acquired. That such infection may be more common than is indicated by the few published cases, is suggested by the recent findings of Wilder $(1952 \mathrm{a}, \mathrm{b})$, who claimed to have demonstrated organisms morphologically indistinguishable from toxoplasma in granulo matous chorio-retinal lesions in 53 eyes of adults; the protozioa were founch in 30 out of 131 eyes with the pathological diagnosis of tuberculosis or pos $\frac{0}{2}$ sible tuberculosis. The lesions in which the organisms were found were very similar and consisted of well-demarcated necrotic areas involving the retina and choroid and frequently the sclera. They were situated posteriorly equatorially, or peripherally, and occasionally more than one lesion was present in a single eye. Sabin and others (1952), however, believe that it is: better to reserve judgment on this interesting communication until the results of serological tests on the patients from whom the eyes were removed are available, or until such time as toxoplasma organisms from similarly affected enucleated eyes, have been demonstrated by animal inoculation.

Further evidence on this subject is presented in this paper, wherein the clinical, serological, and histological findings in a case of chorio-retinitis ing an adult are reported, together with a brief account of the histological examination of 32 cases of granulomatous uveitis.

\section{Case Report}

A male, aged 40, first came under observation in August, 1947, giving an ocular history? that the vision in his left eye had been defective " as long as he could remember". Pre-D sumably this dated from youth. Ten years previously (1937) the vision in this eye had deteriorated further and at that time bilateral antrostomies had been performed. During? the 10 weeks previous to seeking advice he had noticed that the vision in the leff 
eye had again become worse. The general medical history contained nothing significant ; but there was a history of tuberculosis in the family.

Examination.-The right eye was in every respect normal-vision 65 . In the left eye the anterior segment was normal; there was a considerable amount of fine vitreous opacities, and from the upper temporal margin of the disc there extended a large patch of deep choroiditis showing an inflammation of the acute granulomatous type superimposed on an old lesion of long standing. The remainder of the fundus was normal ; there was an absolute field defect corresponding to the chorio-retinal lesion; vision was $6 / 18$.

Diagnosis.-The patient was hospitalized and overhauled with a view to establishing the diagnosis. In general he appeared to be extremely healthy. Bacteriologically the only findings which could be interpreted as possibly pathological were a coagulasepositive staphylococcus in the upper respiratory tract and a streptococcus haemolyticus, type 3 , in the upper respiratory passages and bowel. There was a strongly positive Mantoux reaction to human tuberculin and a weakly positive reaction to bovine tuberculin ; but all other serological tests were negative. Toxoplasma tests were not carried out at this stage. There was no dental infection and $x$ rays of the chest were negative.

The appearance of the eye did not suggest the likelihood of the infection being streptococcal, and, in view of its clinical characteristics, the family history, and the strongly positive Mantoux reaction with the differentiation between the response to human and bovine tuberculin, the case was provisionally labelled tuberculous.

Therapy:-After the acute stage of choroiditis had passed, the patient was given a course of tuberculin injections, starting with minute doses.

Later Developments.--In October, 1947, the lesion was quiet, leaving an atrophic area spreading out widely from the disc: there was still a slight vitreous haze; vision 618 .

The eye remained quiet until August, 1949, when the patient returned with a history that the vision in the left eye had deteriorated suddenly 5 days previously. On examination the anterior segment was again found to be normal, a dense vitreous haze obscured all view of the fundus, central visiun had gone, and hand-movements only were apparent in the peripheral field. With routine treatment the posterior uveitis gradually settled down, and by February, 1950, the vitreous was relatively clear and an immense area of atrophic scarring was seen to occupy the posterior pole involving the macula and embracing the disc, associated with optic atrophy. An extensive relapse of a necrotizing choroiditis had obviously occurred.

The eye remained quiet until September, 1950, when there was again a sudden deterioration of vision, for the first time associated with pain. On examination the ocular tension was found to be raised, the cornea was oedematous with its posterior surface bespattered with keratic precipitates, the iris was heavily vascularized, no view was obtainable behind the pupil, and all perception of light had gone. The clinical picture suggested the presence of a vascular thrombosis of considerable extent. On hospitalization and with intensive local treatment the tension fell and the acute symptoms subsided; but since a considerable degree of discomfort and photophobia remained, the eye was excised in November, 1950. From that time onwards the patient has remained in excellent health.

Summary.-Recurrent posterior uveitis of obscure aetiology, clinically of the granulomatous type, persistently recurring, resulting in loss of perception of light and eventual destruction of the eye.

\section{Pathological Findings}

In November, 1950, the globe was opened horizontally to reveal a chorio-retinal ulceration in the posterior temporal region. Horizontal celloidin sections were cut and stained with haematoxylin and eosin, Van Gieson's stain, and ZiehlNeelsen's stain.

Sections. - There was a mild interstitial inflammatory infiltration of the corneal stroma and small foci of lymphocytes could be seen within the corneal epithelium. Massive 
keratic precipitates were present on the corneal endothelium and the filtration angles we partially occluded by aggregations of inflammatory cells; posterior synechiae we present. Lens normal.

Throughout the uveal tract there was a chronic inflammatory infiltration, severe in the iris, in which nodular foci were present at the pupillary margin, moderate and diffuse the ciliary body and anterior choroid-a delicate cyclitic membrane was present-and severe in the posterior region where there was a well-demarcated area of necrosis on the temporal side. In this region the retina and choroid were fused, being divided only by strip of pigment epithelium and remnants of Bruch's membrane (Figs 1, 2, and 3) ; m the centre of this lesion the retina was necrotic and had completely lost its normal archintecture. There was a mild chronic inflammatory infiltration in the underlying sclera. $\overrightarrow{0}$

Elsewhere the retina showed severe perivascular cuffing and nodular proliferations of endothelial cells, especially around the retinal veins and on the internal limiting membrane

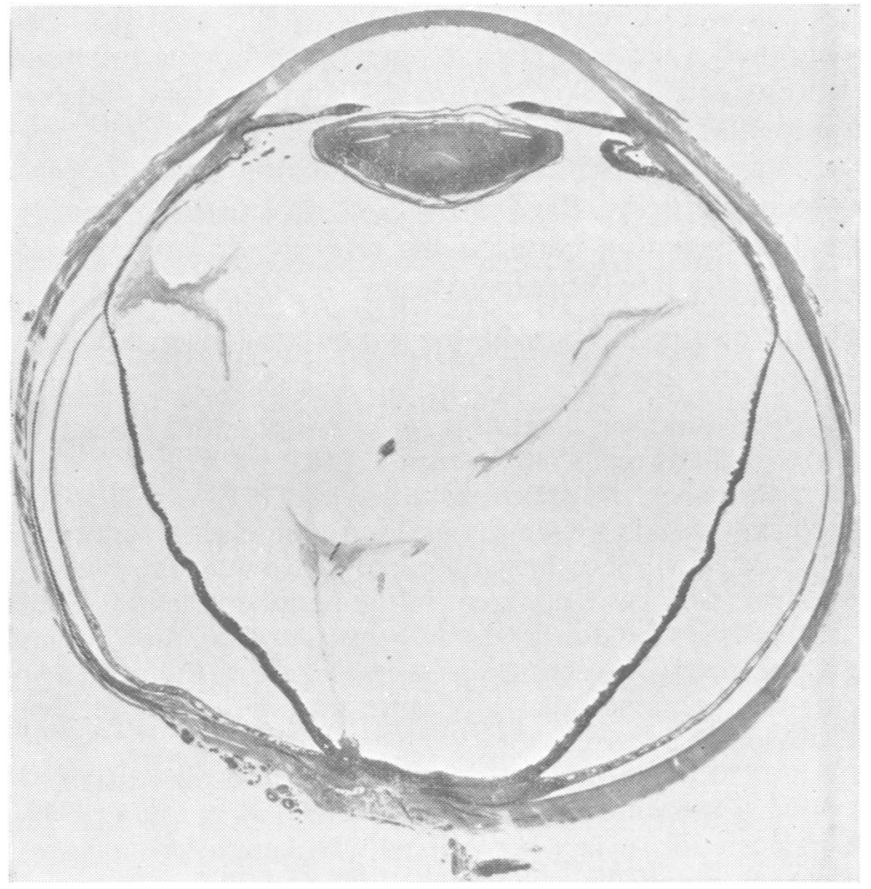

Fig. 1.-Section of globe. Oit the temporal side of the dise there is a well-demarcates area of granulomatous chorio retinitis. See Fig. 2.

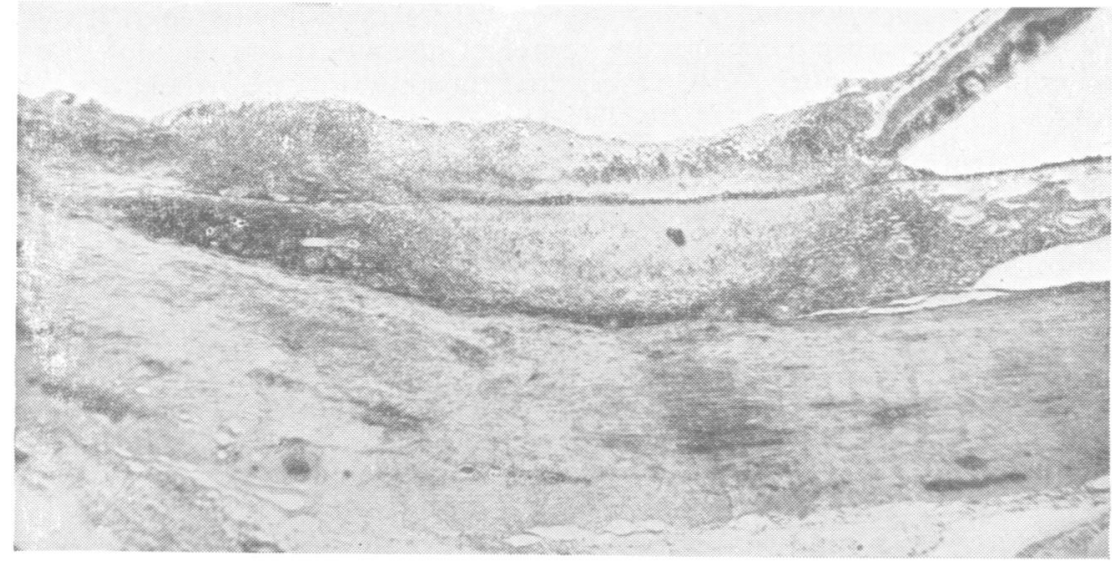

FIG. 2.-High-power view of area of chorio-retinitis. Retina and choroid are fused, being separated only by a layer of pigment epithelium and remnants of Bruch's membrane. The lesion is granulomatous and centrally necrotic. The underlying sclera shows a mild chronic inflammation. Haematoxylin and eosin $\times 22$. 


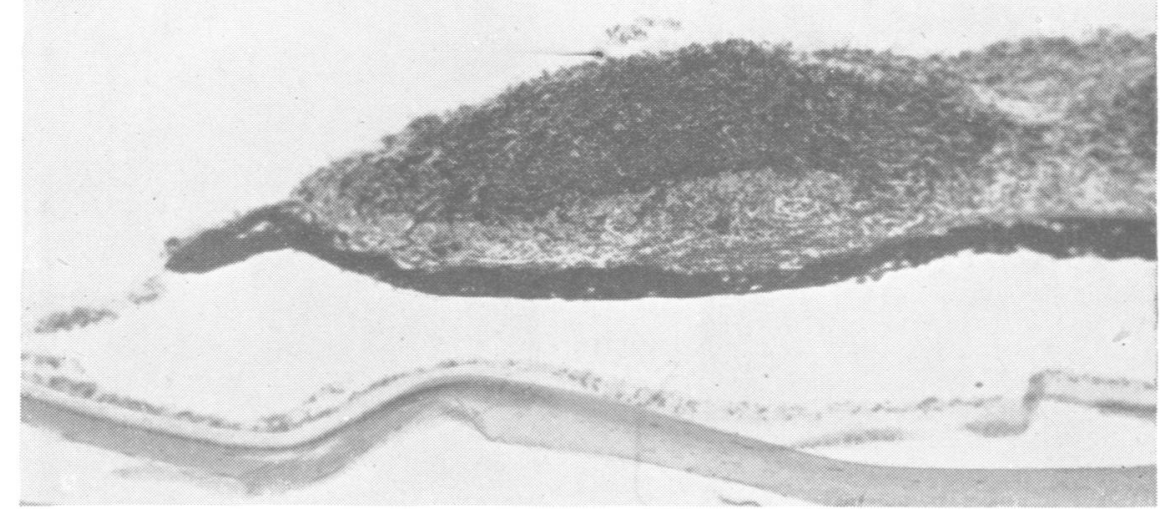

FIG. 3.- Severe chronic inflammatory infiltration of iris with nodular aggregations at pupillary margin. Haematoxylin and eosin $\times 44$.

Some of the smaller retinal vessels had become obliterated by the exuberant tissue proliferation. Scattered eosinophils were present in and around the vessels, but these cells were not prominent ; a few giant cells could be seen in the area of retinal necrosis and in association with the endothelial proliferations elsewhere in the retina, but were not present in the choroid. There was cellular vitreous exudate, particularly dense over the ciliary processes ; the disc was oedematous and showed a severe inflammatory infiltration. Ziehl-Neelsen staining revealed no tubercle bacilli.

The histological diagnosis at this time was chronic granulomatous chorio-uveitis, probably tuberculous in origin. A Middlebrook-Dubos test and agglutination tests for Brucellosis were carried out with negative results.

In August, 1952, in view of the reported findings of Wilder $(1952 \mathrm{a}, \mathrm{b})$ the sections were re-examined 21 months after excision.*

Sections.-Serial sections were cut throughout the necrotic chorio-retinal lesion, periodic acid-Schiff and Heidenhain's iron haematoxylin being used in addition.

Many crescentic structures morphologically indistinguishable from toxoplasma were found in the necrotic area of the retina and in the nodular lesions of the iris (Figs 4, 5,

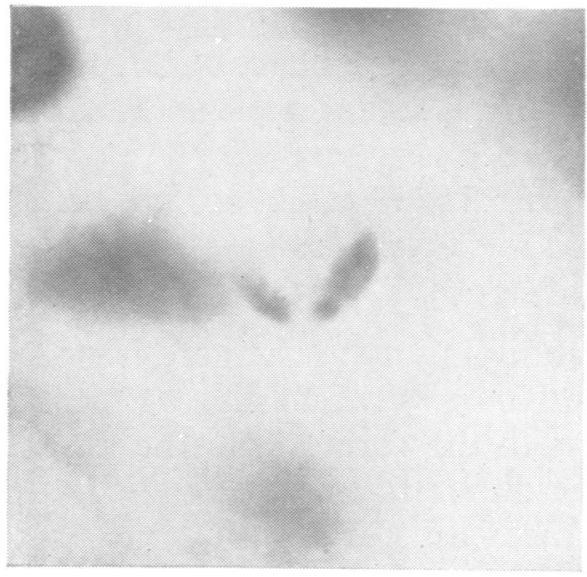

FIG. 4.-V-shaped pair of crescentic structures indistinguishable from toxoplasma. Nuclei localized at adjacent poles. (Authors' case). Haematoxylin and eosin $\times 2560$.

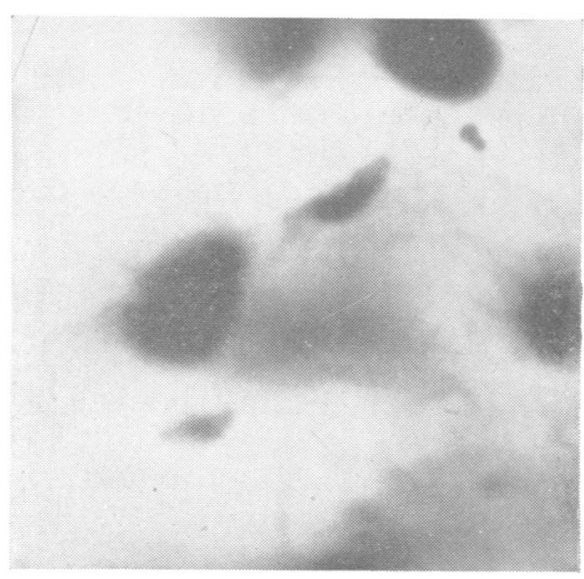

Fig. 5.-Two separate crescentic forms. Lower organism shows a central nucleus and is identical with toxoplasma (Authors' case). Heidenhain's haematoxylin and Masson stain $\times 2540$.

\footnotetext{
* Dr. Helenor Wilder herself selected the slide from this case as a probable example of toxoplasmosis.
} 


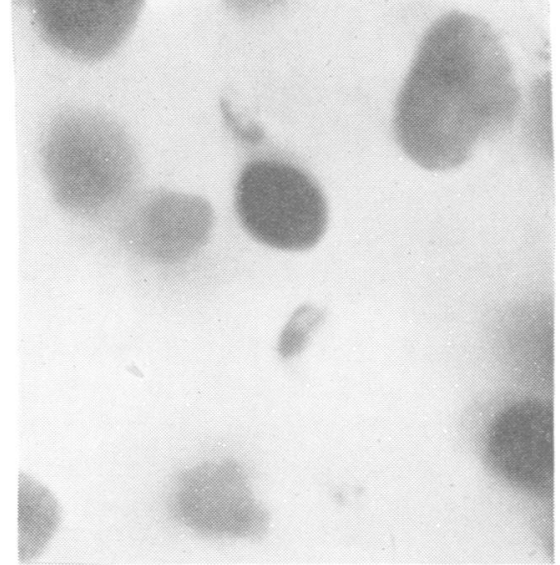

FIG. 6.-Nucleated crescentic form in choroidal granulation tissue (Authors' case). Haematoxylin and eosin $\times 1600$.

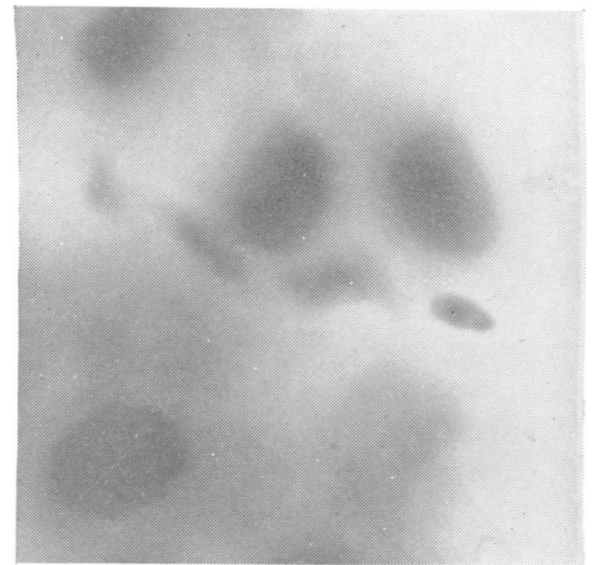

Fig. 7.-Choroidal granulation tissue containing crescent forms, one showing central nucleus (Authors' case). Haematoxylin and eosin $\times 1600$.

6 , and 7). In some of the sections over twenty scattered crescentic forms were found, $\vec{\imath}$ while in others only three or four were observed. Some occurred in pairs, either parallel or in a V-shape, the nuclei being located at the tip of the V. The majority, however, were ${ }^{\Phi}$ isolated, the nuclei being situated either at the middle or at one end, and granules weres sometimes distinguishable in the cytoplasm. A few of the forms were intracellular, but no typical pseudo-cysts or cysts containing toxoplasma were seen. With haematoxylin and eosin the cytoplasm stained faintly blue and the nuclei dark blue. P.A.S. stain was not sufficiently selective to be of use ; Heidenhain's iron haematoxylin was of the greatest value in differentiating the organisms (Smith, 1953). These findings indicated a probable toxoplasmosis, and serological tests were carried out in August, 1952, that is, 2 years after the last relapse of the uveitis. These tests were repeated in October, 1952, and in, January, 1953.

Serological Tests (performed by Professor Beattie, Sheffield University) :

A. Cytoplasm Modifying Dye Test.
1. (18.8.52) Positive up to $1: 160$
2. (18.10.52) Positive up to $1: 68$.
3. (26.1.53) Positive up to $1: 32$.

B. Complement-fixation Test.

1. (18.8.52) Positive $1: 2$.

2. (26.1.53) Negative.
C. Rabbit Skin Test.

1. (18.10.52) Serum neutralized morè than ten and less thane. one hundred skin-tes units.

2. (26.1.53) Ten skin-test units neutra응 lized.

D. Dermal Sensitivity Test to Toxoplasmin.

1. (10.10.52) Strongly positive.

\section{Discussion}

In the interpretation of the cytoplasm modifying dye test there is not yek complete agreement on the levels of the positive titres which may be regardec as significant, and to some extent this is due to the variation in the range of positive results in the healthy populations of different countries. According $\Phi^{D}$ to Sabin and others (1952), however, the titres do not exceed 1:64 in the vast majority of the normal population, and this figure is in accord with $\vec{B}$ the findings of Beverley and Beattie (1952) in Great Britain : 


\begin{tabular}{|c|c|c|c|c|}
\hline Under 10 years & & . & . & $1: 16$ or over. \\
\hline $10-19$ & ․ & . & . & $1: 32$ or over. \\
\hline Over 20 years & $\ldots$ & $\ldots$ & $\ldots$ & $1: 64$ \\
\hline
\end{tabular}

Thus the figure of $1: 160$ obtained in our case, is of possible significance, and becomes even more striking when one considers the rapidly falling titre, which, if due to the removal of the antigenic focus 21 months previously, would suggest a much higher level at the time of the acute phase of the uveitis. Indeed, it is reasonable to suppose that this patient's left eye was the only remaining tissue to have contained viable protozoa ; Weinman (1943) has shown that there is a differential visceral immunity in toxoplasmosis with immunity marked in the lung and feeble in the brain, a phenomenon which has been attributed to a lesser permeability of the cerebral capillaries to antibodies as compared with capillaries elsewhere (Friedemann, 1942). It is possible that the organisms are similarly shielded within the retinal tissues, and that, in the particular case here reported, they had survived only within the excised eye.

With regard to the complement-fixation tests, it has been found that titres as low as $1: 2$ can be specific, in that such sera have also invariably contained significant amounts of cytoplasm-modifying antibody (Sabin and others, 1952). The same authors have established that the complement-fixing antibody appears later and disappears much earlier than the cytoplasmmodifying antibody, so that the findings in our case (a conversion of a positive titre of $1: 2$ to a negative result in $5 \frac{1}{2}$ months) is in accord with a recovery from an acute infection. The rabbit skin tests and the dermal sensitivity tests are confirmatory.

We therefore conclude that the serological results are to be regarded as significant, and that, when these are taken in conjunction with the histological findings, there can be little doubt that the granulomatous chorioretinitis was toxoplasmic in origin. The existence of crescentic forms in the necrotic inflammatory tissue and the absence of pseudo-cysts would appear to indicate an acute phase of the infection, as pointed out by Weinman (1952), while the clinical history of defective vision " as long as he could remember" would suggest a congenital infection or an onset in early childhood. On the other hand, according to Johnson, Fried, Broaddus and Lamfrom (1946), the absence of cerebral calcification indicates an onset after the age of 15 years. The question whether this was a case of congenital infection or of infection acquired post-natally in childhood must, therefore, remain in doubt, but the evidence is in favour of a chronic infection remaining latent in the ocular tissues for many years and recrudescing to give rise to an acute chorio-retinitis in middle age. Thus the findings in this case support in a rather striking way the opinion of Wilder (1952b) that, in adults, granulomatous chorio-retinitis clinically and histologically resembling tuberculosis may be due to toxoplasmosis. Wilder does not state the number of eyes examined, but the fact that the protozoa were demonstrated in 53 cases 
suggests that toxoplasmosis may be a not uncommon cause of chorio-retinitiso in adults. In order to ascertain whether a similar high incidence is to be expected in Great Britain we have examined sections from 32 cases of granulomatous uveitis reported at this Institute since 1948.

None of the 32 cases had the localized type of necrotic lesion described $\overrightarrow{\vec{s}}$ by Wilder as typical of toxoplasmosis. Nevertheless, in about half of the cases (17) a few structures were found resembling, to a greater or lesser extent, crescentic toxoplasma forms, but their appearance was not $\stackrel{\mathbb{\infty}}{\circ}$ sufficiently typical to identify them as protozoa, nor were their isolated क disposition and sparse distribution (never more than five in one case) $\vec{\circ}$ characteristic of toxoplasmosis. That similar crescentic forms were also $\vec{\omega}$ found in cases of post-traumatic granulomatous uveitis (Fig. 8), in which $\frac{0}{0}$. toxoplasmosis was not in question, casts further doubt upon their nature. Furthermore, a solitary, isolated crescentic form with a typical nucleus was ${ }_{0}$ demonstrated in a case of Behcet's syndrome (Fig. 9). It was apparent that degenerate cells undergoing karyolysis and karyorrhexis may often veryo closely resemble toxoplasma pseudo-cysts. In one case in which crescentic $\vec{c}$

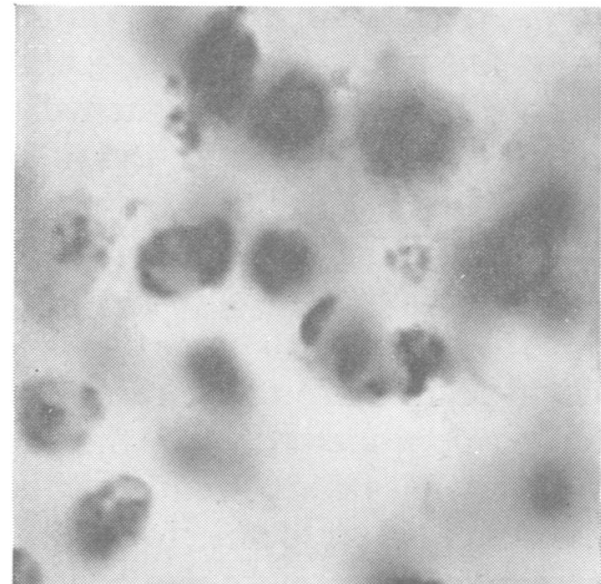

FIG. 8.-Isolated crescentic structure resembling toxoplasma in choroid of a case of traumatic uveitis. Haematoxylin and eosin $\times 1600$.

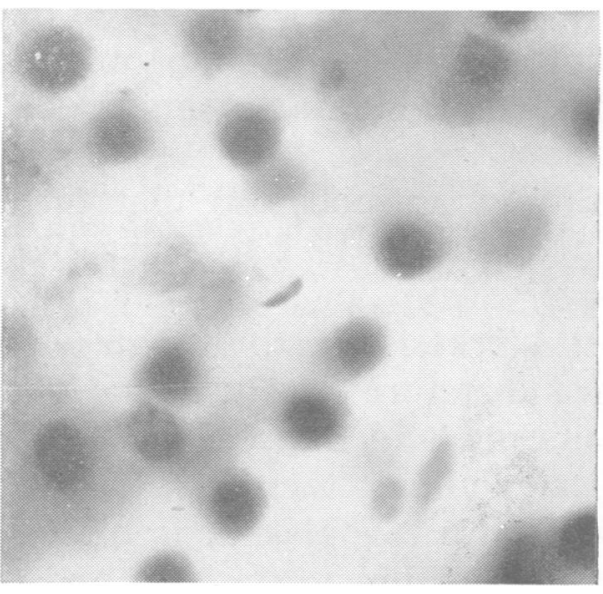

FIG. 9.-Isolated crescentic structure resembling toxoplasma in choroid of a case of Behcet's syndrome. Haematoxylin and eosin $\times 1267$.

structures were found serological tests were carried out (dye test $1: 8 ; \tilde{N}$ complement-fixation test negative).

Thus from this relatively small group of patients it may be concluded that in cases of granulomatous uveitis neither resembling tuberculosis nor readily admitting a diagnosis of toxoplasmosis, histological structures morphologically similar to toxoplasma (probably consisting of cell debris or nuclear debris) may be found.

Further investigation on this important subject is clearly necessary, but in $\stackrel{\mathbb{Q}}{\circ}$ view of the difficulty in interpreting suspicious structures in histological material, we feel that great caution should be exercised before labelling themo 
as toxoplasma. Before regarding the diagnosis as even probable, it is necessary, in our opinion, to have observed quite typical histological formscrescents in pairs being particularly significant-and to have demonstrated positive serological tests.

When sufficient examinations fulfilling the above criteria have accumulated, it may well be that Wilder's suggestion that toxoplasmosis is a not uncommon cause of chorio-retinitis in adults may be generally confirmed. Meanwhile it should be emphasized that there is as yet no certain evidence to show that the case here reported is other than a rarity.

\section{Summary}

(1) A case of recurrent granulomatous chorio-retinitis in an adult is reported, and clinical, histological, and serological evidence is given for regarding the condition as due to toxoplasmosis. It is suggested that the disease was either congenital or acquired in childhood, and that the active chorio-retinitis in adult life was due to a recrudescence of the early infection.

(2) When sections of 32 cases of granulomatous uveitis were examined, about half showed structures resembling toxoplasma forms. However, in some of the cases in which these were found, which included such conditions as post-traumatic uveitis, and Behcet's syndrome, the diagnosis of toxoplasmosis would have been highly improbable. It is, therefore, concluded that these histological appearances probably result from cellular debris or nuclear fragmentation, and that, in the absence of confirmatory serological tests, such findings have a very limited value.

We are indebted to Professor C. P. Beattie and Dr. A. J. K. Beverley who kindly carried out the serological investigations, and to Dr. Peter Hansell for the photographs. Our thanks are also due to Mr. A. McNeil and Mr. V. Elwood for technical assistance.

\section{REFERENCES}

Beverley, J. K. A., and Beattie, C. P. (1952). J. clin. Path., 5,0350.

BOHN, H., and KOCH, E. (1951). Med. Welt, $20,547$.

Campbell, A. M. G., and Clifton, F. (1950). Brain, 73, 281.

FriedemanN, U. (1942). Physiol. Rev., 22, 125.

Guimarães, F. Nery (1943). Mem. Inst. Osw. Cruz, 38, 257.

Johnson, L. V., Fried, N., Broaddus, C. C., and Lamfrom, H. (1946). Arch. Ophthal., Chicago, $36,677$.

Kean, B. H., and Grocott, R. G. (1947). Amer. J. trop. Med., 27, 745.

Magnusson, J. H. (1951). Nord. Med., 45, 344.

NOETZEL, H. (1951). Beitr. path. Anat., 111, 419.

Pinkerton, H., and Henderson, R. G. (1941). J. Amer. med. Ass., 116, 807.

and Weinman, D. (1940). Arch. Path., Chicago, 30, 374.

Plaut, A. (1946). Amer. J. Path., 22, 427.

RiEGER, H. (1951). Klin. Mbl. Augenheilk., 119, 459. (1952). Ibid., 120, 33.

Sabin, A. B. (1942). J. Amer. med. Ass., 116, 801. (1950). Trans. Amer. Acad. Ophthal. Otol. Laryng., 54, 190.

Eichenwald, H., Feldman, H. A., and Jacobs, L. (1952). J. Amer. med. Ass., 150, 1063.

SMITH, C. (1953). British Journal of Ophthalmology, 37, in the press.

SyVerton, J. T., and Slavin, H. B. (1946). J. Amer. med. Ass., 131, 957.

Vail, D., Strong, J. C., and StePhenson, W. V. (1943). Amer. J. Ophthal., 26, 133.

Weinman, D. (1943). J. infect. Dis., 73, 85.

- (1952). Ann. Rev. Microbiol., 6, 281.

Wilder, H. C. (1952a). Arch. Ophthal., Chicago, 47, 425.

- (1952b). Ibid., 48, 127.

WISING, P. (1952). Nord. Med., 47, 563. 